\title{
Propagation of Micro-analyticity for Solutions of Pseudo-differential Equations, I
}

By

\author{
Tetsuji Mıwa
}

\begin{abstract}
Propagation of micro-analyticity is studied for a solution $u$ of a pseudo-differential equation $P(x, D) u=0$ of a certain class whose characteristics are not necessarily of constant multiplicity.
\end{abstract}

\section{Introduction}

Let $P(x, D) u=0$ be a single pseudo-differential equation of finite order defined in a neighborhood of $x_{0}^{*}=\left(x_{0}, \sqrt{-1} \xi_{0} \infty\right)$, a point in the cosphere bundle $\sqrt{-1} S^{*} M$ of a real analytic manifold $M$. This is known to be microlocally equivalent to the simplest equation $\left(\partial / \partial x_{1}\right)^{m} v=0$ provided that $P(x, D)$ is real and with constant multiple characteristics. This fact implies in particular that under the same assumptions microanalyticity or equivalently the zero of a micro-function solution $u$ of $P(x, D) u=0$ propagates along bicharacteristic strips of $P(x, D)$. (See Sato, Kawai and Kashiwara [1]; we also refer to Kawai [2], Hörmander [3] and Andersson [4] for linear differential operators with simple characteristics.)

It is the aim of the present article to extend this result on propagation of micro-analyticity to operators whose characteristics are not necessarily of constant multiplicity. In this part I we establish lemmas on propagation of micro-analyticity of a solution $u$ of a pseudo-differential equation $P(x, D) u=0$ for operators whose principal symbol is a product of real and simply characteristic symbols. As an easy corollary of those lemmas we

Received April 10, 1974. 
deduce a theorem on analyticity of elementary solutions for linear hyperbolic differential operators with real analytic coefficients. A key to our theorem is the existence of good elementary solutions for micro-hyperbolic operators (established in Kashiwara and Kawai [9]).

A part of the results of this paper has been announced in Miwa [10].

In the case of operators with constant coefficients far reaching results have already been established by several authors (Atiyah, Bott and Gårding [5], Andersson [6], [7] and Bernstein [8]). In the subsequent part II we will extend the present results to the wider class of those pseudodifferential operators whose principal symbols are micro-locally contacttransformable to constant-coefficiented symbols so as to comprise the results of these authors.

The author expresses his sincere gratitude to Professor T. Kawai and Professor M. Kashiwara for their kind guidances to their deep theory.

\section{Reductive Pseudo-differential Operators and Their Bicharacteristic Strips}

In this section we introduce a class of pseudo-differential operators and define bicharacteristic strips for them.

Definition 1.1. Reductive pseudo-differential operators. Let $P(x, D)$ be a pseudo-differential operator of finite order defined in a neighborhood of $x_{0}^{*}=\left(x_{0}, \xi_{0} \infty\right)$, a point in the cotangential projective bundle $P^{*} X$ of a complex manifold $X$. Let

$$
\sigma(P)(x, \xi)=p_{1}^{s_{1}}(x, \xi) \ldots p_{r}^{s_{r}}(x, \xi)
$$

be an irreducible decomposition of its principal symbol at $x_{0}^{*}$. We call $P(x, D)$ reductive at $x_{0}^{*}$ if each $\boldsymbol{p}_{j}(x, \xi)$ is simply characteristic, that is, if $d_{(x, \xi)} p_{j}(x, \xi)$ is not parallel to $\sum_{i} \xi_{i} d x_{i}$ at $x_{0}^{*}$. We call $r$ the number of crossing and $s_{1}+\ldots+s_{r}$ the multiplicity.

Examples of reductive symbols are

$$
\xi_{1}^{2}-x_{1}^{2}\left(\xi_{2}^{2}+\ldots+\xi_{n}^{2}\right)
$$

and 


$$
\left(\xi_{1}^{2}-a(x) \xi_{2}^{2}-b(x) \xi_{3}^{2}\right)\left(\xi_{1}^{2}-c(x) \xi_{2}^{2}-d(x) \xi_{3}^{2}\right)
$$

with $a(x), b(x), c(x)$ and $d(x)$ positive when $x$ is real. Both of (1.2) and (1.3) are reductive on their real characteristic varieties.

Definition 1.2. Bicharacteristic strips. Let $P(x, D)$ be as in Definition 1.1. We can define a bicharacteristic strip $B_{j}\left(x_{0}^{*}\right)$ of $\mathbb{p}_{j}(x, \xi)$ through $x_{0}^{*}$ as usual. We call $B_{j}\left(x_{0}^{*}\right)(j=1, \ldots, r)$ bicharacteristic strips of $P(x, D)$ through $x_{0}^{*}$.

\section{A Survey on Micro-hyperbolic Operators}

In this section we recall the results of Kashiwara and Kawai [9], which we need in section 3 .

Let $M$ be a real analytic manifold of dimention $n$ and $X$ be a complex neighborhood of it. We denote by $L$ the cosphere bundle $S_{M}^{*} X=\sqrt{-1} S^{*} M$ and by $\Lambda$ its complex neighborhood. We take a homogeneous local coordinates $\left(x_{1}, \ldots, x_{n}, \sqrt{-1}\left(\xi_{1}, \ldots, \xi_{n}\right) \infty\right)$ of $L$.

Definition 2.1. Partial micro-hyperbolicity. Let $P(x, D)$ be a pseudo-differential operator defined in a neighborhood of $x_{0}^{*}=\left(x_{0}, \sqrt{-}-\overline{1} \xi_{0} \infty\right)$ and $V$ be its characteristic variety, that is, $\{(x, \xi \infty) \in \Lambda ; \sigma(P)(x, \xi)=0\}$. Let $x^{*}+\sqrt{-1}$ vo be a point of $\sqrt{-1} S L=S_{L} \Lambda$. We say that $P(x, D)$ is partially micro-hyperbolic at $x^{*}+\sqrt{-1} v o$ if $x^{*}+\sqrt{-1} v o$ is not contained in the closure of $V-L$ in $\widetilde{L}$, the real monoidal transform of $\Lambda$ with the center $L$ (see Sato, Kawai and Kashiwara [1]). This is equivalent to saying that

$$
\sigma(P)\left(y^{*}+\sqrt{-1} t v\right) \neq 0
$$

when $y^{*}$ is in $L$ and near $x^{*}$ and $0<t \ll 1$.

We denote by $L \hat{\times} L$ the real analytic manifold $\sqrt{-}-1 S^{*}(M \times M)$ $-M \times \sqrt{-1} S^{*} M-\sqrt{-1} S^{*} M \times M$ and we take a homogeneous local coordinates $\left(x_{1}, \ldots, x_{n}, x_{1}^{\prime}, \ldots, x_{n}^{\prime}, \sqrt{-1}\left(\xi_{1}, \ldots, \xi_{n}, \xi_{1}^{\prime}, \ldots, \xi_{n}^{\prime}\right) \infty\right)$ of $L \hat{\times} L$. We identify $L$ with the anti-diagonal set of $L \hat{\times} L$, that is, with $\{(x, x$, $\sqrt{-1}(\xi,-\xi) \infty) \in L \hat{\times} L\}$. 
Now let us consider in a coordinate neighborhood of $L$, where $\xi_{n} \neq 0$. We can use inhomogeneous local coordinates $\left(x_{1}, \ldots, x_{n}, p_{1}, \ldots, p_{n-1}\right)$ of $L$ where $p_{j}=-\frac{\xi_{j}}{\xi_{n}}(j=1, \ldots, n-1)$ and $\left(x_{1}, \ldots, x_{n}, x_{1}^{\prime}, \ldots, x_{n}^{\prime}, p_{1}, \ldots, p_{n-1}, p_{1}^{\prime}\right.$, $\left.\ldots, p_{n}^{\prime}\right)$ of $L \hat{\times} L$ where $p_{j}=-\frac{\xi_{j}}{\xi_{n}}(j=1, \ldots, n-1)$ and $p_{j}^{\prime}=-\frac{\xi_{j}^{\prime}}{\xi_{n}}(j=1, \ldots$, $n) . \quad L$ is identified with $\left\{\left(x_{1}, \ldots, x_{n}, x_{1}, \ldots, x_{n}, p_{1}, \ldots, p_{n-1},-p_{1}, \ldots\right.\right.$, $\left.\left.-p_{n-1}, 1\right)\right\}$ in $L \hat{\times} L$.

Let $\Theta$ be a subbundle of $S_{L}^{*}(L \hat{\times} L)$ induced from the fundamental 1-form on $L \hat{\times} L$. Using the local coordinates $\Theta$ can be written as

$$
\Theta=\left\{\left(x, x, p,-p, 1,\left(d x_{n}-\sum_{j=1}^{n-1} p_{j} d x_{j}-d x_{n}^{\prime}+\sum_{j=1}^{n-1} p_{j} d x_{j}^{\prime}\right) \infty\right)\right\} .
$$

We consider $S^{*} L$ as a subbundle of $S_{L}^{*}(L \hat{\times} L)$ of codimension 1 by the map

$$
\begin{aligned}
& \left(x_{1}, \ldots, x_{n}, p_{1}, \ldots, p_{n-1},\left(\sum_{j=1}^{n} a_{j} d x_{j}+\sum_{j=1}^{n-1} b_{j} d p_{j}\right) \infty\right) \\
& \longmapsto\left(x, x, p,-p, 1,\left(\sum_{j=1}^{n} a_{j} d x_{j}-\sum_{j=1}^{n} a_{j} d x_{j}^{\prime}+\sum_{j=1}^{n-1} b_{j} d p_{j}\right.\right. \\
& \left.\left.+\sum_{j=1}^{n-1} b_{j} d p_{j}^{\prime}+\left(\sum_{j=1}^{n-1} b_{j} p_{j}\right) d p_{n}^{\prime}\right) \infty\right) .
\end{aligned}
$$

Definition 2.2. Canonical map $\boldsymbol{H}$. There is a canonical map $H$ from $S_{L}^{*}(L \hat{\times} L)-\Theta$ to $\sqrt{-1} S L$ induced from the fundamental 1 -form on L. $H$ maps

$$
\left(x, x, p,-p, 1,\left(\sum_{j=1}^{n} a_{j} d x_{j}-\sum_{j=1}^{n} a_{j} d x_{j}^{\prime}+\sum_{j=1}^{n-1} b_{j} d p_{j}+\sum_{j=1}^{n} b_{j} d p_{j}^{\prime}\right) \infty\right)
$$

to

$$
\left(x, p, \sqrt{-1}\left(\sum_{j=1}^{n} b_{j}\left(\partial / \partial x_{j}\right)-\sum_{j=1}^{n-1}\left(a_{n} p_{j}+a_{j}\right)\left(\partial / \partial p_{j}\right)\right) 0\right) .
$$

Lemma 2.1. Let $\theta_{1}, \theta_{2} \in S_{x_{0}}^{*} L$. Then we have

$$
\left\langle H\left(\theta_{1}\right), \theta_{2}\right\rangle=-\left\langle H\left(\theta_{2}\right), \theta_{1}\right\rangle .
$$

If we denote by [, ] the Lagrangean bracket (see Sato, Kawai and Kashiwara [1]),

$$
\left\langle H\left(d a_{1}\right), d a_{2}\right\rangle=\left[a_{1}, a_{2}\right]
$$


is valid.

Now we explain the notion of normal set and conormal set. Let $M$ be a real analytic manifold, $N$ be its submanifold and $G$ be a closed subset of $M$. The normal set of $G$ along $N$ is the intersection of $S_{N} M$ and the closure of $G-N$ in ${ }^{N} \widetilde{M}$ and we denote it by $S_{N} G$. The polar of $S_{N} G$, that is,

$$
\left\{(x, \xi \infty) \in S_{N}^{*} M ;\langle\xi, v\rangle \leqq 0 \text { for any } x+v o \in S_{N} G\right\}
$$

is called the conormal set of $G$ along $N$ and we denote it by $S_{N}^{*} G$.

Definition 2.3. $\mathscr{A}_{\Gamma}$ and $\mathcal{H}_{\Delta}$. Let $x^{*}$ be a point of $L$ and $\Gamma$ be a subset in $S_{L}^{*}(L \hat{\times} L)$ over $x^{*}$. We denote $\mathcal{A}_{\Gamma}$ the set of all germs $K$ of $C_{M M \times N}^{(0, n)}$ at $x^{*}$ such that the fiber over $x^{*}$ of the conormal set of the support of $K$ along $L \subset L \hat{\times} L$ contains a neighborhood of the antipodal set of $\Gamma$. If $\Delta$ is a subset of $\sqrt{-} \overline{1} S_{x * *} L$, then $\mathcal{A}_{H^{-1}(\Delta)}$ is denoted by $\mathcal{H}_{\Delta}$.

Proposition 2.1. Ring structure of $\mathcal{A}_{\Gamma} . \quad A_{\Gamma}$ is a ring by the operation $\left(K_{1}\left(x^{\prime}, x\right) d x, K_{2}\left(x^{\prime}, x\right) d x\right) \longmapsto\left\{\int K_{1}\left(x, x^{\prime \prime}\right) K_{2}\left(x^{\prime \prime}, x^{\prime}\right) d x^{\prime \prime}\right\} d x^{\prime}$ if $\Gamma$ is not empty.

Definition 2.4. $\mathscr{M}_{\Gamma}$. Let $x^{*}$ be a point of $L$ and $\Gamma$ be a subset of $S_{x *}^{*} L$. The set of all germs $u(x)$ of $C_{M}$ at $x^{*}$ satisfying the following condition is denoted by $\mathcal{M}_{\Gamma}$ : the normal set of the support of $\mathfrak{u}(x)$ along $x^{*}$ does not intersect the polar of $\Gamma$.

Proposition 2.2. $\mathscr{M}_{\Gamma}$ is an $\mathcal{A}_{\Gamma}$ module by the operation

$$
\left(K\left(x, x^{\prime}\right) d x^{\prime}, u(x)\right) \longmapsto(K u)(x)=\int K\left(x, x^{\prime}\right) u\left(x^{\prime}\right) d x^{\prime}
$$

Now we can state the main theorem in Kashiwara and Kawai [9].

Theorem 2.1. Existence of a good elementary solution. Let $P(x, D)$ be a single pseudo-differential operator of finite order which is partially micro-hyperbolic at $x^{*}+\sqrt{-1}$ vo. Then there exists a unique elementary solution $E$ of $P$ in $\mathscr{H}_{x^{*}+\sqrt{-1} \text { vo }}$, that is, an element $E$ in $\mathcal{H}_{x+\sqrt{-1} \text { vo }}$ satisfying (2.4)

$$
P E=E P=1 \text {. }
$$




\section{Lemmas on Propagation of Micro-analyticity}

In this section we prove lemmas on propagation of micro-analyticity for solutions of pseudo-differential equations.

The following lemma is another expression of Lemma 3.1.5 and Lemma 3.1.6 of Sato, Kawai and Kashiwara [1].

Lemma 3.1. Normal set of a non-singular hypersurface. Let $\varphi(x)$ be a real analytic function defined in a neighborhood of $x_{0}$, a point of a real analytic minifold $M$. Assume that $d \varphi\left(x_{0}\right) \neq 0$. We can rewrite as

$$
\varphi(x)=g(x)(a(x)+\sqrt{-1} b(x))
$$

where

$$
\begin{aligned}
& g\left(x_{0}\right) \neq 0, \\
& d a\left(x_{0}\right) \neq 0
\end{aligned}
$$

and $b(x) \equiv 0$ on $\{x ; a(x)=0\}$ or $b(x) \equiv 0$.

Then the normal set $S_{M} V$ of $V=\{x \in X ; \varphi(x)=0\}$ is as follows.

i) If $b(x) \equiv 0$, then $\left(S_{M} V\right)_{x_{0}}=\left\{v \in\left(S_{M} X\right)_{x_{0}} ;\left\langle v, d a\left(x_{0}\right)\right\rangle=0\right\}$.

ii) If $b(x) \geqq 0$ on $\{x \in M ; a(x)=0\}$, then

$$
\left(S_{M} V\right)_{x_{0}}=\left\{v \in\left(S_{M} X\right)_{x_{0}} ;\left\langle v, d a\left(x_{0}\right)\right\rangle \leqq 0\right\} .
$$

iii) If $b(x) \leqq 0$ on $\{x \in M ; a(x)=0\}$, then

$$
\left(S_{M} V\right)_{x_{0}}=\left\{v \in\left(S_{M} X\right)_{x_{0}} ;\left\langle v, d a\left(x_{0}\right)\right\rangle \geqq 0\right\} .
$$

iv) Otherwise $\left(S_{M} V\right)_{x_{0}}=\left(S_{M} X\right) x_{0}$.

Let $P(x, D)$ be a pseudo-differential operator defined in a neighborhood of $x_{0}^{*}=(0, \ldots, 0 ; \sqrt{-1}(0, \ldots, 1) \infty) \in L$. We assume that $P(x, D)$ is reductive at $x_{0}^{*}$ and (1.1) is an irreducible decomposition of its principal symbol. Moreover we assume that each $p_{i}(x, \xi)$ is real and partially microhyperbolic at $x_{0}^{*}+\sqrt{-1} v o$ for such an element $v$ that $\langle v, \omega\rangle=0$ where $\omega$ is the fundamental l-form on $L$.

From Lemma 3.1, we have $\left\langle v, d \boldsymbol{p}_{i}(x, \xi)\right\rangle \neq 0$. By a suitable contact transformation of $L$, we can transform $v$ into $\partial / \partial p_{1}$. An associated 
quantized contact transformation transform $P(x, D)$ so that we can take

$$
p_{i}(x, p)=p_{1}-q_{i}\left(x, p^{\prime}\right) \quad(i=1, \ldots, r) .
$$

Therefore $r$-bicharacteristic strips through $x_{0}^{*}$ can be parametrized by $x_{1}$. We denote by $B_{\dot{i}}^{ \pm}$the positive (respectively negative) part, that is to say, the part where $x_{1} \gtrless 0$, of $i$-th bicharacteristic strip through $x_{0}^{*}$.

Our first lemma on propagation of micro-analyticity is as follows.

Lemma 3.2. Assume that $r=2$ and that

$$
\left\{\mathbb{p}_{1}(x, \xi), \mathbb{p}_{2}(x, \xi)\right\} \neq 0
$$

at $x_{0}^{*}$. If $u$ is a microfunction solution of the equation $P(x, D) u=0$ in a neighborhood of $x_{0}^{*}$, and micro-analytic on each $B_{i}^{-}(i=1,2)$, then $u$ is micro-analytic at $x_{0}^{*}$.

For arbitrary $r$ we have only a partial result. We do not know whether the following condition (3.3) is necessary or not.

Lemma 3.3. Assume that

$$
\left\{p_{i}(x, \xi), p_{j}(x, \xi)\right\} \neq 0 \quad \text { for } \quad i \neq i
$$

at $x_{0}^{*}$. Moreover we assume that the skew-symmetric matrix

$$
W=\left(\left\{\boldsymbol{p}_{i}(x, \xi), \boldsymbol{p}_{j}(x, \xi)\right\}\right) \quad i, j=1, \ldots, r
$$

at $x_{0}^{*}$ satisfies the following condition.

(3.3) For any principal minor

$$
W_{I}=\left(\left\{\boldsymbol{p}_{i}(x, \xi), \boldsymbol{p}_{j}(x, \xi)\right\}\right) \quad i, j \in I \subset\{1, \ldots, r\}
$$

such that $\sharp(I) \geqq 2$ and for any vector $\lambda=\left(\lambda_{1}, \ldots, \lambda_{\sharp(I)}\right)$ such that $\lambda_{1} \geqq 0, \ldots$, $\lambda_{\sharp(I) \geqq 0}$

$$
W_{I} \lambda=0
$$

if and only if $\lambda=(0 . \ldots, 0)$.

Then the conclusion of Lemma 3.2 is also valid for $r \geqq 3$.

Proof. We prove Lemma 3.3 under condition (3.3) by the induction 
on $r$. Let

$$
\left(d p_{1}-d q_{i}\right)^{-}=\left\{v \in S_{x_{0}^{*}} L ;\left\langle v, d p_{1}-d q_{i}\right\rangle\langle 0\} .\right.
$$

We also denote by $\left(d p_{1}-d q_{i}\right)+\left(\right.$ respectively $\left.\left(d p_{1}-d q_{i}\right)^{\perp}\right)$ the set

$$
\begin{gathered}
\left\{v \in S_{x_{0}^{*}} L ;\left\langle v, d p_{1}-d q_{i}\right\rangle>0\right\} \quad \text { (respectively } \\
\left.\left\{v \in S_{x_{0}^{*}} L ;\left\langle v, d p_{1}-d q_{i}\right\rangle=0\right\} .\right)
\end{gathered}
$$

$P(x, D)$ is partially micro-hyperbolic at $x_{0}^{*}+\sqrt{-1} v o$ for any $v$ in $\Delta=\bigcap_{i=1}^{r}\left(d p_{1}-d q_{i}\right)^{-}$. Let

$$
\Gamma=H^{-1}(\Delta) \cap S_{x_{0}^{*}}^{*} L=\left(\bigcap_{i=1}^{r} H^{-1}\left(d p_{1}-d q_{i}\right)^{-}\right) \cap S_{x_{0}^{*}}^{*} L
$$

and $\Gamma^{0}$ be its polar in $S_{x_{0}^{*}} L$.

From Lemma 2.1

$$
\left\langle-H\left(d p_{1}-d q_{i}\right), H^{-1}\left(d p_{1}-d q_{i}\right)^{-}\right\rangle=\left\langle d p_{1}-d q_{i},\left(d p_{1}-d q_{i}\right)^{-}\right\rangle\langle 0 .
$$

Hence $\left\{H^{-1}\left(d p_{1}-d q_{i}\right)-\cap S_{x_{0}^{*}}^{*} L\right\}^{0}=-H\left(d p_{1}-d q_{i}\right)$.

Put $v_{i}=-H\left(d p_{1}-d q_{i}\right)$. Then

$$
\begin{gathered}
\Gamma^{0}=v_{1} \bigvee \ldots \bigvee v_{r}=\left\{\sum_{i=1}^{r} \lambda_{i} v_{i} ; \lambda_{i} \geqq 0 \quad i=1, \ldots, r \quad\right. \text { and } \\
\left.\left(\lambda_{1}, \ldots, \lambda_{r}\right) \neq(0, \ldots, 0)\right\}
\end{gathered}
$$

We denote by $G$ the support of $u$. If we can show that

$$
S_{x_{0}^{*}} G \cap \Gamma^{0}=\phi,
$$

then a good elementary solution $E$ for $P(x, D)$ operates on $u$. Hence we have

$$
u=(E P) u=E(P u)=0 .
$$

To prove (3.4), first we recall that micro-analyticity propagates along a bicharacteristic strip if $P(x, D)$ is with constant multiple characteristics.

Let us take a point $x_{1}^{*}$ on $B_{i}^{-}$near $x_{0}^{*}$. From condition (3.2), we may assume that $p_{1}-q_{j}(x, p) \neq 0$ at $x_{1}^{*}$ for $j \neq i$, that is, $P(x, D)$ is of constant multiplicity at $x_{1}^{*}$. If $y_{1}^{*}$ is a point on $V_{i}=\left\{p_{1}-q_{i}(x, p)=0\right\}$ near $x_{1}^{*}$, 
$P(x, D)$ is of constant multiplicity at $y_{1}^{*}$ also. From our assumptions, $u$ is micro-analytic at $x_{1}^{*}$ and hence at $y_{1}^{*}$. If we consider the bicharacteristic strip $B_{i}\left(y_{1}^{*}\right)$ through $y_{1}^{*}$, micro-analyticity of $u$ propagates along $B_{i}\left(y_{1}^{*}\right)$ until when $B_{i}\left(y_{1}^{*}\right)$ meets other characteristic varieties $V_{j}(j \neq i)$.

Micro-analyticity of $u$ propagates along bicharacteristic strips on $V_{j}$ as well as on $V_{i}$, hence from our induction hypothesis, $u$ is still microanalytic if the crossing point is of multiplicity $\leqq r-1$. (Note that from assumption (3.2), a bicharacteristic strip on $V_{k}$ meets other $V_{k^{\prime}}$ only once near $x_{0}^{*}$. Hence if we put $T=\bigcap_{j=1}^{r} V_{j}, u$ is micro-analytic outside of $T \cup B^{+}(T)$ near $x_{0}^{*}$, where $B^{+}(T)=\bigcup_{\substack{x * \in T \\ j=1, \ldots, r}} B_{j}^{+}\left(x^{*}\right)$.

Hence

$$
S_{x_{0}^{*}} G \cap V_{i} \subset S_{x_{0}^{*}} T \vee\left(-v_{i}\right)
$$

It follows that if $S_{x_{0}^{*}} G \cap \Gamma^{0} \cap V_{i} \neq \phi$,

$$
v_{1} \bigvee \ldots \vee v_{r} \cap S_{x_{0}^{*}} T \neq \phi
$$

This implies that for some $\lambda=\left(\lambda_{1}, \ldots, \lambda_{r}\right) \neq(0, \ldots, 0)$ such that $\lambda_{1} \geqq 0, \ldots$, $\lambda_{r} \geqq 0$

$$
\left\langle-\sum_{j=1}^{r} \lambda_{j} H\left(d p_{1}-d q_{j}\right), d p_{1}-d q_{k}\right\rangle=0 \quad(k=1, \ldots, r) .
$$

From Lemma 2.1 this contradicts assumption (3.3).

It is easy to see that if $n=2$ condition (3.2) implies condition (3.3).

Lemma 3.4. Assume that $n=2$ and (3.2) holds, then the conclusion of Lemma 3.2 is valid.

Next we treat the case when $r=2$ and the Poisson bracket vanishes at $x_{0}^{*}$ but not identically. The case when the Poisson bracket vanishes identically will be treated in the subsequent part II.

Lemma 3.5. Assume that $r=2$ and that

$$
\left\{p_{1}(x, \xi), \mathbb{p}_{2}(x, \xi)\right\}=0
$$

at $x_{0}^{*}$. Further assume the following. 
(3.5) There exist positive integers $m_{1}$ and $m_{2}$ such that

$$
\begin{aligned}
& \underbrace{\left\{\boldsymbol{p}_{1},\left\{\boldsymbol{p}_{1}, \ldots,\left\{\boldsymbol{p}_{1}, \boldsymbol{p}_{2}\right\}\right\}\right.}_{m_{1} \text {-times }}\} \neq 0 \\
& \underbrace{\left\{\boldsymbol{p}_{2},\left\{\boldsymbol{p}_{2}, \ldots,\left\{\boldsymbol{p}_{2}, \boldsymbol{p}_{1}\right\}\right\}\right\}}_{m_{2} \text {-times }} \neq 0
\end{aligned}
$$

at $x_{0}^{*}$ and

$$
\left\{\boldsymbol{p}_{1},\left\{\boldsymbol{p}_{1}, \boldsymbol{p}_{2}\right\}\right\}\left\{\boldsymbol{p}_{2},\left\{\boldsymbol{p}_{1}, \boldsymbol{p}_{2}\right\}\right\} \geqq 0
$$

near $x_{0}^{*}$. Then the conclusion of Lemma 3.2 is valid.

Proof. Let $\left(m_{1}\left(x^{*}\right), m_{2}\left(x^{*}\right)\right)$ be the smallest choice of the integers satisfying (3.5) at $x_{0}^{*}$. We prove this lemma by the induction on $m_{1}\left(x^{*}\right)$ and $m_{2}\left(x^{*}\right)$.

As in the proof of Lemma 3.3 it is sufficient to show that

$$
v_{1} \bigvee v_{2} \cap S_{x_{0}^{*}} T=\phi
$$

where

$$
v_{i}=-H\left(d p_{i}\right) \quad i=1,2
$$

and

$$
T=\left\{x^{*} \in L / m_{i}\left(x^{*}\right)=m_{i}\left(x_{0}^{*}\right), \quad i=1,2\right\} .
$$

Let us begin with the case when $m_{1}\left(x_{0}^{*}\right)=m_{2}\left(x_{0}^{*}\right)=2$. Then

$$
T=\left\{p_{1}=p_{2}=\left\{p_{1}, p_{2}\right\}=0\right\}
$$

If (3.7) is not valid, there exists a pair $\left(\lambda_{1}, \lambda_{2}\right) \neq(0,0)$ where $\lambda_{i} \geqq 0(i=1,2)$ such that

$$
-\left\{\lambda_{1} p_{1}+\lambda_{2} p_{2}, p_{i}\right\}=0 \quad i=1,2
$$

and

$$
-\left\{\lambda_{1} p_{1}+\lambda_{2} p_{2},\left\{p_{1}, p_{2}\right\}\right\}=0
$$

at $x_{0}^{*}$. Since we have assumed (3.6), this is a contradiction.

Next we proceed to the case when $m_{1}\left(x_{0}^{*}\right)=2$ and $m_{2}\left(x_{0}^{*}\right)=3$. Then 


$$
T=\left\{\mathbb{p}_{1}=\mathbb{p}_{2}=\left\{\mathbb{p}_{1}, \boldsymbol{p}_{2}\right\}=\left\{\mathbb{p}_{2},\left\{\mathbb{p}_{1}, \mathbb{p}_{2}\right\}\right\}=0\right\} .
$$

Hence if $(3.7)$ is not valid, there exists a pair $\left(\lambda_{1}, \lambda_{2}\right) \neq(0,0)$, where $\lambda_{i} \geqq 0$ $(i=1,2)$ such that

$$
\begin{aligned}
& -\left\{\lambda_{1} \mathbb{p}_{1}+\lambda_{2} \mathbb{p}_{2}, \mathbb{p}_{i}\right\}=0 \quad i=1,2, \\
& -\left\{\lambda_{1} \mathbb{p}_{1}+\lambda_{2} \mathbb{p}_{2},\left\{\mathbb{p}_{1}, \mathbb{p}_{2}\right\}\right\}=0
\end{aligned}
$$

and

$$
-\left\{\lambda_{1} \mathbb{p}_{1}+\lambda_{2} \mathbb{p}_{2},\left\{\mathbb{p}_{2},\left\{\boldsymbol{p}_{1}, \mathbb{p}_{2}\right\}\right\}=0\right.
$$

Since $\left\{\boldsymbol{p}_{1},\left\{\boldsymbol{p}_{1}, \boldsymbol{p}_{2}\right\}\right\} \neq 0,(3.8)$ implies that $\lambda_{1}=0$. Then, since $\left\{\boldsymbol{p}_{2},\left\{\boldsymbol{p}_{2}\right.\right.$, $\left.\left.\left\{\boldsymbol{p}_{1}, \boldsymbol{p}_{2}\right\}\right\}\right\}=0,(3.9)$ implies that $\lambda_{2}=0$. This is a contradiction. In the same way we can proceed with the step of the induction and prove the lemma.

\section{Analyticity of Elementary Solutions}

In this section we define characteristic conoids for operators treated in section 3 and show that the elementary solutions for those operators are analytic outside the characteristic conoids.

Let $P(x, D)$ be a pseudo-differential operator satisfying the conditions stated in section 3 . We assume that $\mathbb{p}_{i}(x, p)(i=1, \ldots, r)$ satisfy the assumptions of one of the lemmas $3.2 \sim 3.5$.

Now we define "bicharacteristic closure" operation for $P(x, D)$.

Definition 4.1. Bicharacteristic closure. Let $x_{0}^{*}$ be a point in a neighborhood where $P(x, D)$ is defined. Let us pursue a bicharacteristic strip $B_{j_{0}}^{+}\left(x_{0}^{*}\right)$ through $x_{0}^{*}$. It may fall across a point $x_{1}^{*}$ of $r\left(x_{1}^{*}\right) \geqq 2$. Then we proceed with pursuing $B_{j_{1}}^{+}\left(x_{1}^{*}\right)$, one of the $r\left(x_{1}^{*}\right)$-bicharacteristic strips through $x_{1}^{*}$. It may fall across again a point $x_{2}^{*}$ of $r\left(x_{2}^{*}\right) \geqq 2$. Then we pursue some $B_{j_{2}}^{+}\left(x_{2}^{*}\right)$ and so on. The union of these bicharacteristic strips is denoted by $B^{+}\left(x_{0}^{*}\right)$ and the union of $B^{+}\left(x_{0}^{*}\right)$ and $x_{0}^{*}$ is called the positive bicharacteristic closure of $x_{0}^{*}$ for $P(x, D)$. Likewise we define $B^{-}\left(x_{0}^{*}\right)$ and the negative bicharacteristic closure. We denote by $B\left(x_{\mathbf{0}}^{*}\right)$ the union of $B^{ \pm}\left(x_{0}^{*}\right)$ and $x_{0}^{*}$ and call it the bicharacteristic closure of $x_{0}^{*}$ 
for $P(x, D)$.

Now let $K \in \mathcal{C}_{M \times M}^{(0, n)}$ be the kernel function of a good elementary solution $E$ of Kashiwara and Kawai [91. Then the following theorem is a trivial consequence of lemmas in section 3 .

Theorem 4.1. Micro-analyticity of elementary solutions. supp $K \subset\left\{\left(x, y, \sqrt{-1}(\xi, \eta) \in \sqrt{-1} S^{*}(M \times M) ; \sigma(P)(x, \xi)=\sigma(P)(y,-\eta)=0\right.\right.$ and $(x, \xi)$ belongs to the positive bicharacteristic closure of $(y,-\eta)\} \cup\{(x, y$, $\left.\sqrt{-1}(\xi, \eta) \in \sqrt{-1} S^{*}(M \times M) ; x=y, \xi=-\eta\right\}$.

Now let $P(x, D)$ be a $m$-th order linear differential operator hyperbolic with respect to the direction $(1, \ldots, 0)$. We assume that $P(x, D)$ is reductive on the real characteristic variety $V_{\boldsymbol{R}}$, and satisfies the assumptions of one of the lemmas $3.2 \sim 3.5$. We define the characteristic conoid for $P(x, D)$.

Definition 4.2. Charactestic conoid. Let $y$ be a point in a neighborhood where $P(x, D)$ is defined. Let

$$
W^{+}(y)=\pi\left(\bigcup_{y^{*} \in \pi^{-1}(y) \cap V_{\boldsymbol{R}}} B^{+}\left(y^{*}\right)\right) \cup y, \quad \text { where } \pi: \sqrt{-1} S^{*} M \longrightarrow M .
$$

This is called the positive characteristic conoid through $y$ for $P(x, D)$. Likewise we define $W^{-}(y)$ and $W(y)$. Moreover we denote by $W^{ \pm}(y)$ (respectively $\hat{W}(y))$ the compliment of the connected component of the compliment of $W^{-}(y)$ (respectively $W(y)$ ) which contains the germ of a set

$$
\left\{x ;|x-y| \ll 1, x_{1}=y_{1}, x \neq y\right\} .
$$

Now the following theorem is a consequence of Theorem 4.1 and the fundamental exact sequence

$$
0 \longrightarrow \mathcal{A} \longrightarrow \mathscr{B} \longrightarrow \pi^{*}(\mathcal{C}) \longrightarrow 0
$$

Theorem 4.2. Analyticity of elementary solutions. Let $E(x, y)$ be a good elementary solution for $P(x, D)$, that is to say $E(x, y)$ is a hyperfunction satisfying

$$
P\left(x, D_{x}\right) E(x, y)=P^{*}\left(y, D_{y}\right) E(x, y)=\delta(x-y)
$$


and

$$
\operatorname{supp} E(x, y) \subset\left\{(x, y) ; x_{1} \geqq y_{1}\right\},
$$

and let $E_{j}(x, y)(j=1, \ldots . n)$ be the elementary solution of the Cauchy problem for $P(x, D)$, that is, $E_{j}(x, y)$ is a hyperfunction satisfying

$$
P\left(x, D_{x}\right) E_{j}(x, y)=0
$$

rind

$$
\left.\left(\partial / \partial x_{1}\right)^{i-1} E_{j}(x, y)\right|_{x_{1}=y_{1}}=\delta_{i j} \delta(x-y) .
$$

Then we have

$$
\begin{array}{ll}
\operatorname{sing} & \operatorname{supp} E(x, y) \subset\left\{(x, y) ; x \in W^{++}(y)\right\}, \\
& \operatorname{supp} E(x, y) \subset\left\{(x, y) ; x \in \hat{V}^{+}(y)\right\}, \\
\operatorname{sing} & \operatorname{supp} E_{j}(x, y) \subset\{(x, y) ; x \in W(y)\}
\end{array}
$$

and

$$
\operatorname{supp} E_{j}(x, y) \subset\{(x, y) ; x \equiv \hat{W}(y)\}
$$

\section{References}

[1] Sato. M., Kawdi, T., and Kashiwara, M.. Microfunction and pseudn-differential equations, Lecture notes in 1Math. 287, Springer, 1973, pp 82-98.

[2] Kawai, T., Construction of local elementary solutions for linear partial differential operator with real analytic cocfficients (I), Publ. R.I.Il.S.. 7. 363-397, (1971).

13] Hormander, L., Uniqueness thcorems and wave front scts for solutions of linear differential equations with analytic coeffirients. Comm. Pure Appl. Math., 24, 671-704, (1971'.

5. Andersson, K. G., Analytic wave front sets for solutions of linear differential equations of principal typc, Trans. Amcr. Math. Soc., 177, 1-27, (1973).

[5] Atiyah, M. F., Bott, R.. and Gårding, L., Lacunas for hyperbolic differential operators with constant cuefficients I, Acta Math., 124. 109-189, (1970).

[6] Andersson, K. G., Propagation of analyticity of solutions of partial ilifferential equations with constant coefficients, Ark. .11at. 8, 277-302, (1971).

[7] Localization and wave fronts, Sem. Goulaouic-Schze'cr/s, Exposé No XXV, (1972-1973).

[8] Bernshtein, I. N., Moluics over a ring of differential operators. Study of the funclamental solutions of equations with constant coefficients, Func. Anal. Appl., 5, 89-101, (1971).

[9] Kashiwara, M., and Kawai, T., On micro-hyperbolic pseudo-differential operators I, (to appear'.

[10] Miwa, T., On micro-analyticity of the clementary solutions of hyperbolic differentidl equations with real analytic coefficients, (to apprar). 
